\title{
The Convergence of Three-Step Iterative Schemes for Generalized $\Phi-$ Hemi-Contractive Mappings and the Comparison of Their Rate of Convergence
}

\author{
Linxin Li $\mathbb{D}$ and Dingping Wu \\ Department of Mathematics, Chengdu University of Information Technology, Chengdu 610225, China \\ Correspondence should be addressed to Linxin Li; 1224020519@qq.com
}

Received 19 April 2021; Accepted 3 June 2021; Published 22 July 2021

Academic Editor: Ljubisa Kocinac

Copyright (C) 2021 Linxin Li and Dingping Wu. This is an open access article distributed under the Creative Commons Attribution License, which permits unrestricted use, distribution, and reproduction in any medium, provided the original work is properly cited.

Charles proved the convergence of Picard-type iteration for generalized $\Phi$-accretive nonself-mappings in a real uniformly smooth Banach space. Based on the theorems of the zeros of strongly $\Phi$-quasi-accretive mappings and fixed points of strongly $\Phi-$ hemicontractions, we extend the results to Noor iterative process and SP iterative process for generalized $\Phi$-hemi-contractive mappings. Finally, we analyze the rate of convergence of four iterative schemes, namely, Noor iteration, iteration of Corollary 2, SP iteration, and iteration of Corollary 4.

\section{Introduction and Preliminaries}

In 2009, Charles [1] proved the convergence of Picard-type iteration for generalized $\Phi$-accretive nonself-mappings in a real uniformly smooth Banach space. In this paper, we consider that the Noor iteration process and SP iteration process will be extended from the results of Charles [1].

In [2], Noor et al. proved the convergence of Noor iteration to fixed point of a pseudocontractive self-map defined in a real uniformly smooth Banach space. In [3], Qin and Yao showed the weak convergence of a Mann-like algorithm for nonexpansive and accretive operators. In [4], the author considers some generalized nonexpansive mappings on convex metric spaces and gives some sufficient and necessary conditions for a Noor-type iteration to approximate a common fixed point of an infinite family of uniformly quasi-sup $\left(f_{n}\right)$-Lipschitzian mappings and an infinite family of $g_{n}$-expansive mappings in convex metric spaces.

In 1953, the most general Mann iterative scheme now studied is the following: $x_{0} \in K$,

$$
x_{n+1}=\left(1-c_{n}\right) x_{n}+c_{n} T x_{n}, \quad n=0,1,2, \ldots,
$$

where $\left\{c_{n}\right\}_{n=1}^{\infty} \subset(0,1)$ is a real sequence satisfying appropriate conditions. In 1974, Ishikawa [5] introduced the Ishikawa iteration process as follows: for a convex subset $D$ of a Banach space $E$ and a mapping $T$ from $D$ into itself, for any given $x_{0} \in D$, the sequence $\left\{x_{n}\right\}$ in $D$ is defined by

$$
\left\{\begin{array}{l}
x_{n+1}=\left(1-\alpha_{n}\right) x_{n}+\alpha_{n} T y_{n}, \\
y_{n}=\left(1-\beta_{n}\right) x_{n}+\beta_{n} T x_{n}, \quad n \geq 0,
\end{array}\right.
$$

where $\left\{\alpha_{n}\right\}$ and $\left\{\beta_{n}\right\}$ are two sequences in $[0,1]$ satisfying the conditions $0 \leq \alpha_{n} \leq \beta_{n} \leq 1$, for all $n, \lim _{n \rightarrow \infty} \beta_{n}=0$ and $\sum_{n=0}^{\infty} \alpha_{n} \beta_{n}=\infty$.

In 2000, Noor [6, 7] gave the following three-step iterative schemes for solving nonlinear operator equations in uniformly smooth Banach spaces.

Let $D$ be a nonempty convex subset of $E$ and let $T: D \longrightarrow D$ be a mapping. For a given $\left\{x_{n}\right\}$ in $D$, compute the sequence $\left\{x_{n}\right\}_{n=0}^{\infty}$ by the iterative schemes:

$$
\left\{\begin{array}{l}
x_{n+1}=\left(1-\alpha_{n}\right) x_{n}+\alpha_{n} T y_{n}, \\
y_{n}=\left(1-\beta_{n}\right) x_{n}+\beta_{n} T z_{n}, \\
z_{n}=\left(1-\gamma_{n}\right) x_{n}+\gamma_{n} T x_{n}, \quad n \geq 0,
\end{array}\right.
$$


which is called the Noor iterative process, where $\left\{\alpha_{n}\right\},\left\{\beta_{n}\right\}$, and $\left\{\gamma_{n}\right\}$ are three real sequences in [0,1] satisfying some certain conditions.

In 2011, Phuengrattana and Suantai defined the SP iteration schemes [8] as follows.

Let $D$ be a nonempty convex subset of $E$ and let $T: D \longrightarrow D$ be a mapping. For a given $\left\{x_{n}\right\}$ in $D$, define the sequence $\left\{x_{n}\right\}_{n=0}^{\infty}$ by

$$
\left\{\begin{array}{l}
x_{n+1}=\left(1-\alpha_{n}\right) y_{n}+\alpha_{n} T y_{n}, \\
y_{n}=\left(1-\beta_{n}\right) z_{n}+\beta_{n} T z_{n}, \\
z_{n}=\left(1-\gamma_{n}\right) x_{n}+\gamma_{n} T x_{n}, \quad n \geq 0
\end{array}\right.
$$

where $\left\{\alpha_{n}\right\},\left\{\beta_{n}\right\}$, and $\left\{\gamma_{n}\right\}$ are three real sequences in $[0,1]$ satisfying some certain conditions.

Definition 1 (see [1]). Given a gauge function $\phi$, the mapping $J_{\phi}: E \longrightarrow 2^{E^{*}}$ defined by

$$
J_{\phi} x:=\left\{u^{*} \in E^{*}:\left\langle x, u^{*}\right\rangle=\|x\|\left\|u^{*}\right\| ;\left\|u^{*}\right\|=\phi(\|x\|)\right\}
$$

is called the duality map with gauge function $\phi$, where $E$ is any normed space. In the particular case, $\phi(t)=t$, the duality map $J=J_{\phi}$ is called the normalized duality map.

Proposition 1 (see [9, 10]). If a Banach space $E$ has a uniformly Gateaux differentiable norm, then $J: E \longrightarrow E^{*}$ is uniformly continuous on bounded subsets of E from the strong topology of $E$ to the weak* topology of $E^{*}$.

Definition 2 (see [11]). Let $E$ be an arbitrary real normed linear space. A mapping $T: D(T) \subseteq E \longrightarrow E$ is called strongly hemi-contractive if $F(T) \neq \varnothing$, and there exists $t>1$ such that, for all $r>0$,

$$
\left\|x-x^{*}\right\| \leq\left\|(1+r)\left(x-x^{*}\right)-r t\left(T x-x^{*}\right)\right\|,
$$

holds for all $x \in D(T), x^{*} \in F(T)$. If $t=1$, then $T$ is called hemi-contractive. Finally, $T$ is called generalized $\Phi$-hemicontractive with strictly increasing continuous function $\Phi:[0, \infty) \longrightarrow[0, \infty)$ such that $\Phi(0)=0$ if, for all $x \in D(T), x^{*} \in F(T) \neq \varnothing$, there exists $j\left(x-x^{*}\right) \in J(x-$ $\left.x^{*}\right)$ such that

$$
\left\langle(I-T) x-(I-T) x^{*}, j\left(x-x^{*}\right)\right\rangle \geq \Phi\left(\left\|x-x^{*}\right\|\right) .
$$

It follows from inequality (7) that $T$ is generalized $\Phi$-hemi-contractive if and only if

$$
\left\langle T x-x^{*}, j\left(x-x^{*}\right)\right\rangle \leq\left\|x-x^{*}\right\|^{2}-\Phi\left(\left\|x-x^{*}\right\|\right), \quad \forall n \geq 0 .
$$

Definition 3 (see $[1,12]$ ). Let $N(T)=\{x \in E: T x=0\} \neq \varnothing$. The mapping $T: D(T) \subseteq E \longrightarrow E$ is called generalized $\Phi$-quasi-accretive with strictly increasing continuous function $\Phi:[0, \infty) \longrightarrow[0, \infty)$ such that $\Phi(0)=0$ if, for all $x \in E, x^{*} \in N(T) \neq \varnothing$, there exists $j\left(x-x^{*}\right) \in J\left(x-x^{*}\right)$ such that

$$
\left\langle T x-T x^{*}, j\left(x-x^{*}\right)\right\rangle \geq \Phi\left(\left\|x-x^{*}\right\|\right) .
$$

Definition 4 (see $[13,14]$ ). A mapping $T: D \longrightarrow D$ is called generalized Lipschitz if there exists a constant $L>0$ such that $\|T x-T y\| \leq L(1+\|x-y\|), \forall x, y \in D$.

Proposition 2 (see [1]). If $F(T)=\{x \in E$ : Tx $=x\} \neq \varnothing$, the mapping $T: E \longrightarrow E$ is strongly hemi-contractive if and only if $(I-T)$ is strongly quasi-accretive, $T$ is strongly $\phi$-hemicontractive if and only if $(I-T)$ is strongly $\phi$-quasi-accretive, and $T$ is generalized $\Phi$-hemi-contractive if and only if ( $I-$ $T$ ) is generalized $\Phi$-quasi-accretive.

Proposition 3 (see [1]). Let E be a uniformly smooth real Banach space, and let $J: E \longrightarrow 2^{E^{*}}$ be a normalized duality mapping. Then,

$$
\|x+y\|^{2} \leq\|x\|^{2}+2\langle y, J(x+y)\rangle,
$$

for all $x, y \in E$.

Proposition 4 (see [1]). Let $\left\{\lambda_{n}\right\}$ and $\left\{\gamma_{n}\right\}$ be sequences of nonnegative numbers and $\left\{\alpha_{n}\right\}$ be a sequence of positive numbers satisfying the conditions $\sum_{n=1}^{\infty} \alpha_{n}=\infty$ and $\left(\gamma_{n} / \alpha_{n}\right) \longrightarrow 0$, as $n \longrightarrow \infty$. Let the recursive inequality

$$
\lambda_{n+1} \leq \lambda_{n}-\alpha_{n} \psi\left(\lambda_{n}\right)+\gamma_{n}, \quad n=1,2, \ldots
$$

be given, where $\psi:[0, \infty) \longrightarrow[0, \infty)$ is strictly increasing continuous function such that it is positive on $(0, \infty)$ and $\psi(0)=0$. Then, $\lambda_{n} \longrightarrow 0$, as $n \longrightarrow \infty$.

\section{Main Results}

In this section, we will consider to extend the result of Charles [1] to the Noor iteration process and SP iteration process under the following assumptions.

First, we extend the result of Charles [1] to the Noor iteration process.

Theorem 1. Suppose D is a nonempty closed convex subset of a real uniformly smooth Banach space E. Suppose $T: D \longrightarrow D$ is a bounded generalized Lipschitz $\Phi$-hemicontractive mapping and $x^{*} \in F(T) \neq \varnothing$. For arbitrary $x_{0} \in D,\left\{x_{n}\right\}$ is a Noor iterative sequence defined by (3), where $\left\{\alpha_{n}\right\},\left\{\beta_{n}\right\},\left\{\gamma_{n}\right\} \subseteq[0,1], \lim _{n \longrightarrow \infty} \alpha_{n}=\lim _{n \longrightarrow \infty} \beta_{n}=0, \gamma_{n}=$ $o\left(\beta_{n}\right)$, and $\sum \alpha_{n}=\infty$. Then, there exists a constant $d_{0}>0$ such that if $0<\alpha_{n}, \beta_{n}, \gamma_{n} \leq d_{0},\left\{x_{n}\right\}$ converges strongly to the unique fixed point $x^{*}$ of $T$.

Proof. Since $T: D \longrightarrow D$ is a bounded generalized Lipschitz $\Phi$-hemi-contractive mapping, there exists a strictly increasing continuous function $\Phi:[0, \infty) \longrightarrow[0, \infty)$ with $\Phi(0)=0$ such that

$$
\left\langle(I-T) x-(I-T) x^{*}, j\left(x-x^{*}\right)\right\rangle \geq \Phi\left(\left\|x-x^{*}\right\|\right),
$$

i.e., 


$$
\left\langle T x-x^{*}, j\left(x-x^{*}\right)\right\rangle \leq\left\|x-x^{*}\right\|^{2}-\Phi\left(\left\|x-x^{*}\right\|\right), \quad \forall n \geq 0,
$$

for any $x, y \in D, x^{*} \in F(T)$ and exist a constant $L>0$ such that

$$
\|T x-T y\| \leq L(1+\|x-y\|), \quad \forall x, y \in D .
$$

Let $r$ be sufficiently large such that $x_{1} \in B_{r}\left(x^{*}\right)$. Define $G:=\overline{B_{r}\left(x^{*}\right)} \cap D$. Then, since $T$ is bounded, we have that $(I-T)(G)$ is bounded.

As $j$ is uniformly continuous on bounded subsets of $E$, for $\varepsilon_{0}$ : $=(\Phi(r / 4) / 40 L(1+2 r))$, there exists a $\delta:(r / 2)>\delta>0$ such that $x, y \in D(T),\|x-y\|<\delta$ implies $\|j(x)-j(y)\|<\varepsilon_{0}$. Set $d_{0}=\min \{(\delta / 2[L+\quad(1+2 L) r])$, $\left.\sqrt{\left(\Phi(r / 4) / 8 r^{2}\right)}\right\}$.

Claim 1. $\left\{x_{n}\right\}$ is bounded.

Suffices to show that $x_{n}$ is in $G$ for all $n \geq 1$. The proof is by induction. By our assumption, $x_{1} \in G$. Suppose $x_{n} \in G$. We prove that $x_{n+1} \in G$. Assume for contradiction that $x_{n+1} \notin G$. Then, since $x_{n+1} \in D, \forall n \geq 1$, we have that $\left\|x_{n+1}-x^{*}\right\|>r$. We have the following estimates:

$$
\begin{aligned}
\left\|\left(x_{n}-x^{*}\right)-\left(z_{n}-x^{*}\right)\right\| & \leq \gamma_{n}\left\|x_{n}-T x_{n}\right\| \\
& \leq \gamma_{n}\left(\left\|x_{n}-x^{*}\right\|+\left\|T x_{n}-x^{*}\right\|\right) \\
& \leq d_{0}[L+(1+L) r]<\delta, \\
\left\|z_{n}-x^{*}\right\| & =\left\|\left(1-\gamma_{n}\right) x_{n}+\gamma_{n} T x_{n}-x^{*}\right\| \\
& \leq\left(1-\gamma_{n}\right)\left\|x_{n}-x^{*}\right\|+\gamma_{n}\left\|T x_{n}-x^{*}\right\| \\
& \leq\left(1-\gamma_{n}\right)\left\|x_{n}-x^{*}\right\|+\gamma_{n} L\left(1+\left\|x_{n}-x^{*}\right\|\right) \\
& \leq r+d_{0} L(1+r) \\
& \leq 2 r .
\end{aligned}
$$

Using (15), we obtain

$$
\left\|T z_{n}-x^{*}\right\| \leq L\left(1+\left\|z_{n}-x^{*}\right\|\right) \leq L(1+2 r) .
$$

Using (16), we obtain

$$
\begin{aligned}
\left\|y_{n}-x^{*}\right\| & =\left\|\left(1-\beta_{n}\right) x_{n}+\beta_{n} T z_{n}-x^{*}\right\| \\
& \leq\left(1-\beta_{n}\right)\left\|x_{n}-x^{*}\right\|+\beta_{n}\left\|T z_{n}-x^{*}\right\| \\
& \leq r+d_{0} L\left(1+\left\|z_{n}-x^{*}\right\|\right) \\
& \leq 2 r
\end{aligned}
$$

and

$$
\begin{aligned}
\left\|\left(y_{n}-x^{*}\right)-\left(x_{n}-x^{*}\right)\right\| & \leq \beta_{n}\left\|T z_{n}-x_{n}\right\| \\
& \leq \beta_{n}\left(\left\|T z_{n}-x^{*}\right\|+\left\|x_{n}-x^{*}\right\|\right) \\
& \leq d_{0} \cdot[L+(1+2 L) r]<\delta
\end{aligned}
$$

Using (17), we obtain

$$
\begin{aligned}
\left\|T y_{n}-x^{*}\right\| & \leq L\left(1+\left\|y_{n}-x^{*}\right\|\right) \\
& \leq L(1+2 r) .
\end{aligned}
$$

Using (19), we obtain

$$
\begin{aligned}
\left\|\left(x_{n+1}-x^{*}\right)-\left(x_{n}-x^{*}\right)\right\| & \leq \alpha_{n}\left\|T y_{n}-x_{n}\right\| \\
& \leq \alpha_{n}\left(\left\|T y_{n}-x^{*}\right\|+\left\|x_{n}-x^{*}\right\|\right) \\
& \leq d_{0}[L+(1+2 L) r]<\delta .
\end{aligned}
$$

Then,

$$
\begin{aligned}
\left\|x_{n}-x^{*}\right\| & \geq\left\|x_{n+1}-x^{*}\right\|-\alpha_{n}\left\|T y_{n}-x_{n}\right\| \\
& \geq r-d_{0}[L+(1+2 L) r] \\
& \geq \frac{r}{2} \\
\left\|z_{n}-x^{*}\right\| & \geq\left\|x_{n}-x^{*}\right\|-\gamma_{n}\left\|T x_{n}-x_{n}\right\| \\
& \geq \frac{r}{2}-d_{0}[L+(1+L) r] \\
& \geq \frac{r}{4} \\
\left\|y_{n}-x^{*}\right\| & \geq\left\|x_{n}-x^{*}\right\|-\beta_{n}\left\|T z_{n}-x_{n}\right\| \\
& \geq \frac{r}{2}-d_{0}[L+(1+2 L) r] \\
& \geq \frac{r}{4}
\end{aligned}
$$

Therefore,

$$
\begin{array}{r}
\left\|j\left(x_{n+1}-x^{*}\right)-j\left(x_{n}-x^{*}\right)\right\|<\varepsilon_{0}, \\
\left\|j\left(x_{n}-x^{*}\right)-j\left(y_{n}-x^{*}\right)\right\|<\varepsilon_{0}, \\
\left\|j\left(x_{n}-x^{*}\right)-j\left(z_{n}-x^{*}\right)\right\|<\varepsilon_{0} .
\end{array}
$$

Using Proposition 3 and the above formulas, we obtain 


$$
\begin{aligned}
\left\|z_{n}-x^{*}\right\|^{2}= & \left\|\left(1-\gamma_{n}\right) x_{n}+\gamma_{n} T x_{n}-x^{*}\right\|^{2} \\
= & \left\|\left(1-\gamma_{n}\right) x_{n}-\left(1-\gamma_{n}\right) x^{*}+\gamma_{n} T x_{n}-\gamma_{n} x^{*}\right\|^{2} \\
\leq & \left(1-\gamma_{n}\right)^{2}\left\|x_{n}-x^{*}\right\|^{2}+2 \gamma_{n}\left\langle T x_{n}-x^{*}, j\left(x_{n}-x^{*}\right)\right\rangle \\
& +2 \gamma_{n}\left\langle T x_{n}-x^{*}, j\left(z_{n}-x^{*}\right)-j\left(x_{n}-x^{*}\right)\right\rangle \\
\leq & r^{2}+2 \gamma_{n} L(1+r) \varepsilon_{0}, \\
\left\|y_{n}-x^{*}\right\|^{2}= & \left\|\left(1-\beta_{n}\right) x_{n}+\beta_{n} T z_{n}-x^{*}\right\|^{2} \\
\leq & \left(1-\beta_{n}\right)^{2}\left\|x_{n}-x^{*}\right\|^{2}+2 \beta_{n}\left\langle T z_{n}-x^{*}, j\left(z_{n}-x^{*}\right)\right\rangle \\
& +2 \beta_{n}\left\langle T z_{n}-x^{*}, j\left(y_{n}-x^{*}\right)-j\left(x_{n}-x^{*}\right)\right\rangle \\
& +2 \beta_{n}\left\langle T z_{n}-x^{*}, j\left(x_{n}-x^{*}\right)-j\left(z_{n}-x^{*}\right)\right\rangle \\
\leq & \left(1-\beta_{n}\right)^{2}\left\|x_{n}-x^{*}\right\|^{2}+2 \beta_{n}\left[\left\|z_{n}-x^{*}\right\|^{2}-\Phi\left(\left\|z_{n}-x^{*}\right\|\right)\right] \\
& +2 \beta_{n}\left\|T z_{n}-x^{*}\right\|\left\|j\left(y_{n}-x^{*}\right)-j\left(x_{n}-x^{*}\right)\right\| \\
& +2 \beta_{n}\left\|T z_{n}-x^{*}\right\|\left\|j\left(x_{n}-x^{*}\right)-j\left(z_{n}-x^{*}\right)\right\| \\
\leq & \left(1-\beta_{n}\right)^{2} r^{2}+2 \beta_{n}\left[\left\|z_{n}-x^{*}\right\|^{2}-\Phi\left(\left\|z_{n}-x^{*}\right\|\right)\right] \\
& +4 \beta_{n} L(1+2 r) \varepsilon_{0}, \\
\left\|x_{n+1}-x^{*}\right\|^{2}= & \left\|\left(1-\alpha_{n}\right) x_{n}+\alpha_{n} T y_{n}-x^{*}\right\|^{2} \\
\leq & \left(1-\alpha_{n}\right)^{2}\left\|x_{n}-x^{*}\right\|^{2}+2 \alpha_{n}\left\langle T y_{n}-x^{*}, j\left(y_{n}-x^{*}\right)\right\rangle \\
& +2 \alpha_{n}\left\langle T y_{n}-x^{*}, j\left(x_{n}-x^{*}\right)-j\left(y_{n}-x^{*}\right)\right\rangle \\
& +2 \alpha_{n}\left\langle T y_{n}-x^{*}, j\left(x_{n+1}-x^{*}\right)-j\left(x_{n}-x^{*}\right)\right\rangle \\
\leq & \left(1-\alpha_{n}\right)^{2}\left\|x_{n}-x^{*}\right\|^{2}+2 \alpha_{n}\left[\left\|y_{n}-x^{*}\right\|^{2}-\Phi\left(\left\|y_{n}-x^{*}\right\|\right)\right] \\
& +2 \alpha_{n}\left\|T y_{n}-x^{*}\right\|\left\|j\left(x_{n}-x^{*}\right)-j\left(y_{n}-x^{*}\right)\right\| \\
& +2 \alpha_{n}\left\|T y_{n}-x^{*}\right\|\left\|j\left(x_{n+1}-x^{*}\right)-j\left(x_{n}-x^{*}\right)\right\| \\
\leq & \left(1-\alpha_{n}\right)^{2} r^{2}+4 \alpha_{n} L(1+2 r) \varepsilon_{0} \\
& +2 \alpha_{n}\left[\left\|y_{n}-x^{*}\right\|^{2}-\Phi\left(\left\|y_{n}-x^{*}\right\|\right)\right] . \\
&
\end{aligned}
$$

Substitute (23) into (24) and then substitute (24) into (25); since $0<\alpha_{n}, \beta_{n}, \gamma_{n} \leq d_{0}$ and $d_{0}=\min \{(\delta / 2[L+$ $\left.(1+2 L) r]), \sqrt{\left(\Phi(r / 4) / 8 r^{2}\right)}\right\}$, we have

$$
\begin{aligned}
\left\|x_{n+1}-x^{*}\right\|^{2} \leq & \left(1-\alpha_{n}\right)^{2} r^{2}+4 \alpha_{n} L(1+2 r) \varepsilon_{0} \\
& +2 \alpha_{n}\left[\left(1-\beta_{n}\right)^{2} r^{2}+4 \beta_{n} L(1+2 r) \varepsilon_{0}\right] \\
& +2 \alpha_{n}\left\{2 \beta_{n}\left[r^{2}+2 \gamma_{n} L(1+r) \varepsilon_{0}-\Phi\left(\frac{r}{4}\right)\right]-\Phi\left(\frac{r}{4}\right)\right\} \\
\leq & r^{2}+2 \alpha_{n}\left[2 \beta_{n}^{2} r^{2}+10 L(1+2 r) \varepsilon_{0}-2 \beta_{n} \Phi\left(\frac{r}{4}\right)-\Phi\left(\frac{r}{4}\right)\right] \\
\leq & r^{2}+2 \alpha_{n}\left[\frac{\Phi(r / 4)}{2}-2 \beta_{n} \Phi\left(\frac{r}{4}\right)-\Phi\left(\frac{r}{4}\right)\right] \\
\leq & r^{2},
\end{aligned}
$$

i.e., $\left\|x_{n+1}-x^{*}\right\| \leq r$, a contradiction. Therefore, $x_{n+1} \in G$. Thus, by induction, $\left\{x_{n}\right\}$ is bounded. Then, $\left\{y_{n}\right\},\left\{z_{n}\right\}$, $\left\{T x_{n}\right\},\left\{T y_{n}\right\}$, and $\left\{T z_{n}\right\}$ are also bounded.

Claim 2. $x_{n} \longrightarrow x^{*}$.

Let $A_{n}=\left\|j\left(x_{n+1}-x^{*}\right)-j\left(x_{n}-x^{*}\right)\right\|, B_{n}=\| j\left(x_{n}-x^{*}\right)$ $-j\left(y_{n}-x^{*}\right)\left\|, C_{n}=\right\| j\left(z_{n}-x^{*}\right)-j\left(x_{n}-x^{*}\right) \|$. Note that $x_{n+1}-x_{n} \longrightarrow 0, x_{n}-y_{n} \longrightarrow 0$, and $z_{n}-x_{n} \longrightarrow 0 \quad$ as $n \longrightarrow \infty$, and hence, by the uniform continuity of $j$ on bounded subsets of $E$, we have that

$$
\begin{aligned}
& A_{n} \longrightarrow 0, \\
& B_{n} \longrightarrow 0, \\
& C_{n} \longrightarrow 0, \text { as } n \longrightarrow \infty .
\end{aligned}
$$

Let $M_{1}=\max \left\{\sup \left\|x_{n}-x^{*}\right\|, \sup \left\|T x_{n}-x^{*}\right\|, \sup \| T y_{n}-\right.$ $x^{*} \|$, sup $\left.\left\|T z_{n}-x^{*}\right\|\right\}$; by (24)-(26), we obtain that 


$$
\begin{aligned}
\left\|z_{n}-x^{*}\right\|^{2} \leq & \left(1-\gamma_{n}\right)^{2}\left\|x_{n}-x^{*}\right\|^{2}+2 \gamma_{n} M_{1} C_{n} \\
\leq & \left\|x_{n}-x^{*}\right\|^{2}+2 \gamma_{n} M_{1} C_{n} \\
\left\|y_{n}-x^{*}\right\|^{2} \leq & \left(1-\beta_{n}\right)^{2}\left\|x_{n}-x^{*}\right\|^{2}+2 \beta_{n}\left[\left\|z_{n}-x^{*}\right\|^{2}-\Phi\left(\left\|z_{n}-x^{*}\right\|\right)\right] \\
& +2 \beta_{n} M_{1} C_{n}+2 \beta_{n} M_{1} B_{n}
\end{aligned}
$$

and

$$
\begin{aligned}
\left\|x_{n+1}-x^{*}\right\|^{2} \leq & \left(1-\alpha_{n}\right)^{2}\left\|x_{n}-x^{*}\right\|^{2}+2 \alpha_{n}\left[\left\|y_{n}-x^{*}\right\|^{2}-\Phi\left(\left\|y_{n}-x^{*}\right\|\right)\right] \\
& +2 \alpha_{n}\left\|T y_{n}-x^{*}\right\|\left\|j\left(x_{n+1}-x^{*}\right)-j\left(x_{n}-x^{*}\right)\right\| \\
& +2 \alpha_{n}\left\|T y_{n}-x^{*}\right\|\left\|j\left(x_{n}-x^{*}\right)-j\left(y_{n}-x^{*}\right)\right\| \\
\leq & \left(1-\alpha_{n}\right)^{2}\left\|x_{n}-x^{*}\right\|^{2}+2 \alpha_{n} M_{1} A_{n}+2 \alpha_{n} M_{1} B_{n} \\
& +2 \alpha_{n}\left[\left\|y_{n}-x^{*}\right\|^{2}-\Phi\left(\left\|y_{n}-x^{*}\right\|\right)\right] .
\end{aligned}
$$

$$
\begin{aligned}
\left\|x_{n+1}-x^{*}\right\|^{2} \leq & \left(1-\alpha_{n}\right)^{2}\left\|x_{n}-x^{*}\right\|^{2}+2 \alpha_{n} M_{1} A_{n}+2 \alpha_{n} M_{1} B_{n} \\
& +2 \alpha_{n}\left[\left(1+\beta_{n}^{2}\right)\left\|x_{n}-x^{*}\right\|^{2}-\Phi\left(\left\|y_{n}-x^{*}\right\|\right)\right] \\
& +2 \alpha_{n}\left\{2 \beta_{n}\left[2 \gamma_{n} M_{1} C_{n}-\Phi\left(\left\|z_{n}-x^{*}\right\|\right)+M_{1} C_{n}+M_{1} B_{n}\right]\right\} \\
\leq & \left\|x_{n}-x^{*}\right\|^{2}+2 \alpha_{n}\left[\frac{\alpha_{n}}{2} M_{1}^{2}+\beta_{n}^{2} M_{1}^{2}-\Phi\left(\left\|y_{n}-x^{*}\right\|\right)\right] \\
& +2 \alpha_{n} M_{1} A_{n}+2 \alpha_{n} M_{1} B_{n}+4 \alpha_{n} \beta_{n} M_{1} C_{n}+4 \alpha_{n} \beta_{n} M_{1} B_{n} \\
& +2 \alpha_{n}\left[4 \beta_{n} \gamma_{n} M_{1} C_{n}-2 \beta_{n} \Phi\left(\left\|z_{n}-x^{*}\right\|\right)\right] \\
\leq & \left\|x_{n}-x^{*}\right\|^{2}+2 \alpha_{n}\left[Q_{n}-2 \beta_{n} \Phi\left(\left\|z_{n}-x^{*}\right\|\right)-\Phi\left(\left\|y_{n}-x^{*}\right\|\right)\right],
\end{aligned}
$$

where $Q_{n}=\left(\alpha_{n} / 2\right) M_{1}^{2}+\beta_{n}^{2} M_{1}^{2}+4 \beta_{n} \gamma_{n} M_{1} C_{n}+2 \beta_{n} M_{1} C_{n}+$ $2 \beta_{n} M_{1} B_{n}+M_{1} A_{n}+M_{1} B_{n} \longrightarrow 0$ as $n \longrightarrow \infty$.

Set inf $\left(\Phi\left(\left\|z_{n}-x^{*}\right\|\right) / \Phi\left(\left\|x_{n}-x^{*}\right\|\right)+1\right)=W$; since $\Phi$ is a strictly increasing continuous function, then $W$ exists. Thus,

$$
\Phi\left(\left\|z_{n}-x^{*}\right\|\right) \geq W \Phi\left(\left\|x_{n}-x^{*}\right\|\right)+W \geq W \Phi\left(\left\|x_{n}-x^{*}\right\|\right) .
$$

Set inf $\left(\Phi\left(\left\|y_{n}-x^{*}\right\|\right) / \Phi\left(\left\|x_{n}-x^{*}\right\|\right)+1\right)=N$; since $\Phi$ is a strictly increasing continuous function, then $N$ exists. Thus,

$$
\Phi\left(\left\|y_{n}-x^{*}\right\|\right) \geq N \Phi\left(\left\|x_{n}-x^{*}\right\|\right)+N \geq N \Phi\left(\left\|x_{n}-x^{*}\right\|\right) .
$$

Then,

$$
\begin{aligned}
\left\|x_{n+1}-x^{*}\right\|^{2} & \leq\left\|x_{n}-x^{*}\right\|^{2}+2 \alpha_{n}\left[Q_{n}-2 \beta_{n} \Phi\left(\left\|z_{n}-x^{*}\right\|\right)-\Phi\left(\left\|y_{n}-x^{*}\right\|\right)\right] \\
& \leq\left\|x_{n}-x^{*}\right\|^{2}-2 \alpha_{n}\left(2 \beta_{n} W-N\right) \Phi\left(\left\|x_{n}-x^{*}\right\|\right)+2 \alpha_{n} Q_{n} .
\end{aligned}
$$

Let $\lambda_{n}=\left\|x_{n}-x^{*}\right\|^{2}, S_{n}=2\left(2 \beta_{n} W-N\right)$, and $\rho_{n}=2 \alpha_{n} Q_{n}$; then, from inequality (34), we obtain that $\lambda_{n+1} \leq$ $\lambda_{n}-\alpha_{n} S_{n} \Phi\left(\lambda_{n}\right)+\rho_{n}$, where $\left(\rho_{n} / \alpha_{n} S_{n}\right) \longrightarrow 0$ as $n \longrightarrow \infty$.
Therefore, the conclusion of the theorem follows from Proposition 4. Uniqueness of $x^{*}$ is derived from the definition of $T$. 
By Definition 4, we know that the generalized Lipschitz mapping is the extension of nonexpansive mapping, so the following corollary follows trivially.

Corollary 1. Suppose D is a nonempty closed convex subset of a real uniformly smooth Banach space E. Suppose $T: D \longrightarrow D$ is a bounded generalized $\Phi$-hemi-contractive mapping and $x^{*} \in F(T) \neq \varnothing$. For arbitrary $x_{0} \in D,\left\{x_{n}\right\}$ is a Noor iterative sequence defined by (3), where $\left\{\alpha_{n}\right\}$, $\left\{\beta_{n}\right\},\left\{\gamma_{n}\right\} \subseteq[0,1], \lim _{n \longrightarrow \infty} \alpha_{n}=\lim _{n \longrightarrow \infty} \beta_{n}=0, \gamma_{n}=o\left(\beta_{n}\right)$, and $\sum \alpha_{n}=\infty$. Then, there exists a constant $d_{0}>0$ such that if $0<\alpha_{n}, \beta_{n}, \gamma_{n} \leq d_{0},\left\{x_{n}\right\}$ converges strongly to the unique fixed point $x^{*}$ of $T$.

The following corollary follows trivially from Definition 2 and Definition 3.

Corollary 2. Suppose $E$ is a real uniformly smooth Banach space. Suppose $T: E \longrightarrow E$ is a bounded generalized $\Phi$-accretive mapping and the solution $x^{*}$ of the equation $T x=0$ exists. For arbitrary $x_{0} \in E,\left\{x_{n}\right\}$ in $E$ is defined as

$$
\left\{\begin{array}{l}
x_{n+1}=\left(1-\alpha_{n}\right) x_{n}-\alpha_{n} T y_{n}, \\
y_{n}=\left(1-\beta_{n}\right) x_{n}-\beta_{n} T z_{n}, \\
z_{n}=\left(1-\gamma_{n}\right) x_{n}-\gamma_{n} T x_{n}, \quad n \geq 0,
\end{array}\right.
$$

where $\quad\left\{\alpha_{n}\right\},\left\{\beta_{n}\right\},\left\{\gamma_{n}\right\} \subseteq[0,1], \quad \lim _{n \longrightarrow \infty} \alpha_{n}=\lim _{n \longrightarrow \infty}$ $\beta_{n}=0, \gamma_{n}=o\left(\beta_{n}\right)$, and $\sum \alpha_{n}=\infty$. Then, there exists a constant $d_{0}>0$ such that if $0<\alpha_{n}, \beta_{n}, \gamma_{n} \leq d_{0},\left\{x_{n}\right\}$ converges strongly to the unique solution $T x=0$.

Now, we extend the result of Charles [1] to the SP iteration process as follows.

Theorem 2. Suppose D is a nonempty closed convex subset of a real uniformly smooth Banach space E. Suppose $T: D \longrightarrow D$ is a bounded generalized Lipschitz $\Phi$-hemicontractive mapping and $x^{*} \in F(T) \neq \varnothing$. For arbitrary $x_{0} \in D,\left\{x_{n}\right\}$ is a SP iterative sequence defined by (4), where $\left\{\alpha_{n}\right\},\left\{\beta_{n}\right\},\left\{\gamma_{n}\right\} \subseteq[0,1], \lim _{n \longrightarrow \infty} \alpha_{n}=\lim _{n \longrightarrow \infty} \beta_{n}=0, \quad \gamma_{n}=$ $o\left(\beta_{n}\right)$, and $\sum \gamma_{n}=\infty$. Then, there exists a constant $d_{0}>0$ such that if $0<\alpha_{n}, \beta_{n}, \gamma_{n} \leq d_{0},\left\{x_{n}\right\}$ converges strongly to the unique fixed point $x^{*}$ of $T$.

Proof. Since T: $D \longrightarrow D$ is a bounded generalized Lipschitz $\Phi$-hemi-contractive mapping, there exists a strictly increasing continuous function $\Phi:[0, \infty) \longrightarrow[0, \infty)$ with $\Phi(0)=0$ such that

$$
\left\langle(I-T) x-(I-T) x^{*}, j\left(x-x^{*}\right)\right\rangle \geq \Phi\left(\left\|x-x^{*}\right\|\right),
$$

i.e.,

$$
\left\langle T x-x^{*}, j\left(x-x^{*}\right)\right\rangle \leq\left\|x-x^{*}\right\|^{2}-\Phi\left(\left\|x-x^{*}\right\|\right), \quad \forall n \geq 0,
$$

for any $x, y \in D$ and $x^{*} \in F(T)$, and there exist a constant $L>0$ such that

$$
\|T x-T y\| \leq L(1+\|x-y\|), \quad \forall x, y \in D .
$$

Let $r$ be sufficiently large such that $x_{1} \in B_{r}\left(x^{*}\right)$. Define $G:=\overline{B_{r}\left(x^{*}\right)} \cap D$. Then, since $T$ is bounded, we have that $(I-T)(G)$ is bounded.

As $j$ is uniformly continuous on bounded subsets of $E$, for $\varepsilon_{0}$ : $=(\Phi(r / 4) / 6[L+3 r(1+L)])$, there exists a $\delta:(r / 4)>\delta>0$ such that $x, y \in D(T),\|x-y\|<\delta$ implies $\|j(x)-j(y)\|<\varepsilon_{0}$. Set $d_{0}=(\delta / 2[L+(1+L) 3 r])$.

Claim 3. $\left\{x_{n}\right\}$ is bounded.

Suffices to show that $x_{n}$ is in $G$ for all $n \geq 1$. The proof is by induction. By our assumption, $x_{1} \in G$. Suppose $x_{n} \in G$. We prove that $x_{n+1} \in G$. Assume for contradiction that $x_{n+1} \notin G$. Then, since $x_{n+1} \in D, \forall n \geq 1$, we have that $\left\|x_{n+1}-x^{*}\right\|>r$. We have the following estimates:

$$
\begin{aligned}
\left\|\left(x_{n}-x^{*}\right)-\left(z_{n}-x^{*}\right)\right\| & \leq \gamma_{n}\left\|x_{n}-T x_{n}\right\| \\
& \leq \gamma_{n}\left(\left\|x_{n}-x^{*}\right\|+\left\|T x_{n}-x^{*}\right\|\right) \\
& \leq d_{0}[L+(1+L) r]<\delta, \\
\left\|z_{n}-x^{*}\right\| & =\left\|\left(1-\gamma_{n}\right) x_{n}+\gamma_{n} T x_{n}-x^{*}\right\| \\
& \leq\left(1-\gamma_{n}\right)\left\|x_{n}-x^{*}\right\|+\gamma_{n}\left\|T x_{n}-x^{*}\right\| \\
& \leq\left(1-\gamma_{n}\right)\left\|x_{n}-x^{*}\right\|+\gamma_{n} L\left(1+\left\|x_{n}-x^{*}\right\|\right) \\
& \leq r+d_{0} L(1+r) \\
& \leq 2 r .
\end{aligned}
$$

Using (39), we obtain

$$
\begin{aligned}
\left\|T z_{n}-z_{n}\right\| & \leq\left\|T z_{n}-x^{*}\right\|+\left\|z_{n}-x^{*}\right\| \\
& \leq L+(1+L) 2 r .
\end{aligned}
$$

Using (40), we obtain

$$
\begin{aligned}
\left\|\left(z_{n}-x^{*}\right)-\left(y_{n}-x^{*}\right)\right\| & \leq \beta_{n}\left\|z_{n}-T z_{n}\right\| \\
& \leq \beta_{n}\left(\left\|z_{n}-x^{*}\right\|+\left\|T z_{n}-x^{*}\right\|\right) \\
& \leq \beta_{n}[2 r+L(1+2 r)]<\delta, \\
\left\|y_{n}-x^{*}\right\| & =\left\|\left(1-\beta_{n}\right) z_{n}+\beta_{n} T z_{n}-x^{*}\right\| \\
& \leq\left(1-\beta_{n}\right)\left\|z_{n}-x^{*}\right\|+\beta_{n}\left\|T z_{n}-x^{*}\right\| \\
& \leq 2 r+d_{0} L(1+2 r) \\
& \leq 3 r .
\end{aligned}
$$

Using (41), we obtain 


$$
\begin{aligned}
\left\|\left(y_{n}-x^{*}\right)-\left(x_{n+1}-x^{*}\right)\right\| & \leq \alpha_{n}\left(\left\|y_{n}-x^{*}\right\|+\left\|T y_{n}-x^{*}\right\|\right) \\
& \leq \alpha_{n}\left[\left\|y_{n}-x^{*}\right\|+L\left(1+\left\|y_{n}-x^{*}\right\|\right)\right] \\
& \leq d_{0}[3 r+L(1+3 r)]<\delta \\
\left\|T y_{n}-y_{n}\right\| & =\left\|\left(T y_{n}-x^{*}\right)-\left(y_{n}-x^{*}\right)\right\| \\
& \leq L\left(1+\left\|y_{n}-x^{*}\right\|\right)+\left\|y_{n}-x^{*}\right\| \\
& \leq L+(1+L) 3 r .
\end{aligned}
$$

Then,

$$
\begin{aligned}
\left\|\left(y_{n}-x^{*}\right)\right\| & \geq\left\|x_{n+1}-x^{*}\right\|-\alpha_{n}\left\|T y_{n}-y_{n}\right\| \\
& \geq r-d_{0}[L+(1+L) 3 r] \\
& \geq \frac{r}{2} \\
\left\|z_{n}-x^{*}\right\| & \geq\left\|y_{n}-x^{*}\right\|-\gamma_{n}\left\|T z_{n}-z_{n}\right\| \\
& \geq \frac{r}{2}-d_{0}[L+(1+L) 2 r] \\
& \geq \frac{r}{4} .
\end{aligned}
$$

Therefore,

$$
\begin{array}{r}
\left\|j\left(x_{n+1}-x^{*}\right)-j\left(y_{n}-x^{*}\right)\right\|<\varepsilon_{0}, \\
\left\|j\left(y_{n}-x^{*}\right)-j\left(z_{n}-x^{*}\right)\right\|<\varepsilon_{0}, \\
\left\|j\left(z_{n}-x^{*}\right)-j\left(x_{n}-x^{*}\right)\right\|<\varepsilon_{0} .
\end{array}
$$

Using Proposition 3 and the above formulas, we obtain

$$
\begin{aligned}
\left\|z_{n}-x^{*}\right\|^{2}= & \left\|\left(1-\gamma_{n}\right) x_{n}+\gamma_{n} T x_{n}-x^{*}\right\|^{2} \\
= & \left\|\left(1-\gamma_{n}\right) x_{n}-\left(1-\gamma_{n}\right) x^{*}+\gamma_{n} T x_{n}-\gamma_{n} x^{*}\right\|^{2} \\
\leq & \left\|x_{n}-x^{*}\right\|^{2}-2 \gamma_{n}\left\langle(I-T) x_{n}, j\left(x_{n}-x^{*}\right)\right\rangle \\
& +2 \gamma_{n}\left\langle(I-T) x_{n}, j\left(z_{n}-x^{*}\right)-j\left(x_{n}-x^{*}\right)\right\rangle \\
\leq & \left\|x_{n}-x^{*}\right\|^{2}-2 \gamma_{n} \Phi\left(\left\|x_{n}-x^{*}\right\|\right) \\
& +2 \gamma_{n}[L+(1+L) r] \varepsilon_{0} \\
\leq & r^{2}+2 \gamma_{n}[L+(1+L) r] \varepsilon_{0},
\end{aligned}
$$

$$
\begin{aligned}
\left\|y_{n}-x^{*}\right\|^{2}= & \left\|\left(1-\beta_{n}\right) z_{n}+\beta_{n} T z_{n}-x^{*}\right\|^{2} \\
= & \left\|\left(z_{n}-x^{*}\right)-\beta_{n}(I-T) z_{n}\right\|^{2} \\
\leq & \left\|z_{n}-x^{*}\right\|^{2}-2 \beta_{n}\left\langle(I-T) z_{n}, j\left(z_{n}-x^{*}\right)\right\rangle \\
& \quad-2 \beta_{n}\left\langle(I-T) z_{n}, j\left(y_{n}-x^{*}\right)-j\left(z_{n}-x^{*}\right)\right\rangle \\
\leq & \left\|z_{n}-x^{*}\right\|^{2}-2 \beta_{n} \Phi\left(\left\|z_{n}-x^{*}\right\|\right) \\
& +2 \beta_{n}\left\|z_{n}-T z_{n}\right\|\left\|j\left(y_{n}-x^{*}\right)-j\left(z_{n}-x^{*}\right)\right\| \\
\leq & \left\|z_{n}-x^{*}\right\|^{2}-2 \beta_{n} \Phi\left(\left\|z_{n}-x^{*}\right\|\right) \\
& +2 \beta_{n}[L+(1+L) 2 r] \varepsilon_{0},
\end{aligned}
$$

$$
\begin{aligned}
\left\|x_{n+1}-x^{*}\right\|^{2}= & \left\|\left(1-\alpha_{n}\right) y_{n}+\alpha_{n} T y_{n}-x^{*}\right\|^{2} \\
\leq & \left\|y_{n}-x^{*}-\alpha_{n}(I-T) y_{n}\right\|^{2} \\
\leq & \left\|y_{n}-x^{*}\right\|^{2}-2 \alpha_{n}\left\langle(I-T) y_{n}, j\left(y_{n}-x^{*}\right)\right\rangle \\
& \quad-2 \alpha_{n}\left\langle(I-T) y_{n}, j\left(x_{n+1}-x^{*}\right)-j\left(y_{n}-x^{*}\right)\right\rangle \\
\leq & \left\|y_{n}-x^{*}\right\|^{2}-2 \alpha_{n} \Phi\left(\left\|y_{n}-x^{*}\right\|\right) \\
& +2 \alpha_{n}\left\|y_{n}-T y_{n}\right\|\left\|j\left(x_{n+1}-x^{*}\right)-j\left(y_{n}-x^{*}\right)\right\| \\
\leq & \left\|y_{n}-x^{*}\right\|^{2}-2 \alpha_{n} \Phi\left(\left\|y_{n}-x^{*}\right\|\right) \\
& +2 \alpha_{n}[L+(1+L) 3 r] \varepsilon_{0} .
\end{aligned}
$$

Substitute (45) into (46) and then substitute (46) into (47); since $0<\alpha_{n}, \beta_{n}, \gamma_{n} \leq d_{0}$ and $d_{0}=(\delta / 2[L+(1+L) 3 r])$, we have

$$
\begin{aligned}
\left\|x_{n+1}-x^{*}\right\|^{2} \leq & r^{2}+2 \gamma_{n}[L+(1+L) r] \varepsilon_{0}-2 \beta_{n} \Phi\left(\frac{r}{4}\right) \\
& +2 \beta_{n}[L+(1+L) 2 r] \varepsilon_{0}-2 \alpha_{n} \Phi\left(\frac{r}{2}\right) \\
& +2 \alpha_{n}[L+(1+L) 3 r] \varepsilon_{0} \\
\leq & r^{2}+2 \alpha_{n}\left\{[L+(1+L) 3 r] \varepsilon_{0}+\frac{\beta_{n}}{\alpha_{n}}[L+(1+L) 2 r] \varepsilon_{0}\right\} \\
& +2 \alpha_{n}\left\{\frac{\gamma_{n}}{\alpha_{n}}[L+(1+L) r] \varepsilon_{0}-\Phi\left(\frac{r}{2}\right)-\frac{\beta_{n}}{\alpha_{n}} \Phi\left(\frac{r}{4}\right)\right\} \\
\leq & r^{2}+2 \alpha_{n}\left[\frac{\Phi(r / 4)}{2}-\Phi\left(\frac{r}{4}\right)-\frac{\beta_{n}}{\alpha_{n}} \Phi\left(\frac{r}{4}\right)\right] \\
\leq & r^{2},
\end{aligned}
$$


i.e., $\left\|x_{n+1}-x^{*}\right\| \leq r$, a contradiction. Therefore, $x_{n+1} \in G$. Thus, by induction, $\left\{x_{n}\right\}$ is bounded. Then, $\left\{y_{n}\right\},\left\{z_{n}\right\},\left\{T x_{n}\right\},\left\{T y_{n}\right\}$, and $\left\{T z_{n}\right\}$ are also bounded.

Claim 4. $x_{n} \longrightarrow x^{*}$.

Let

$j\left(y_{n}-x^{*}\right)\left\|, B_{n}=\right\| j\left(y_{n}-x^{*}\right)-$

$j\left(z_{n}-x^{*}\right)\left\|, C_{n}=\right\| j\left(z_{n}-x^{*}\right)-j\left(x_{n}-x^{*}\right) \| ; \quad$ note that $x_{n+1}-y_{n} \longrightarrow 0, y_{n}-z_{n} \longrightarrow 0$, and $z_{n}-x_{n} \longrightarrow 0$, as $n \longrightarrow \infty$, and hence, by the uniform continuity of $j$ on bounded subsets of $E$, we have that

$$
\begin{aligned}
& A_{n} \longrightarrow 0, \\
& B_{n} \longrightarrow 0, \\
& C_{n} \longrightarrow 0, \text { as } n \longrightarrow \infty
\end{aligned}
$$

Let $\quad M_{1}=\max \left\{\sup \left\|y_{n}-T y_{n}\right\|\right.$, sup $\left\|z_{n}-T z_{n}\right\|$, $\sup \left\|x_{n}-T x_{n}\right\|$, sup $\left.\left\|T z_{n}-x^{*}\right\|\right\}$; by (46)-(48), we obtain that

$$
\begin{aligned}
& \left\|z_{n}-x^{*}\right\|^{2} \leq\left\|x_{n}-x^{*}\right\|^{2}-2 \gamma_{n} \Phi\left(\left\|x_{n}-x^{*}\right\|\right)+2 \gamma_{n} M_{1} C_{n}, \\
& \left\|y_{n}-x^{*}\right\|^{2} \leq\left\|z_{n}-x^{*}\right\|^{2}-2 \beta_{n} \Phi\left(\left\|z_{n}-x^{*}\right\|\right)+2 \beta_{n} M_{1} B_{n},
\end{aligned}
$$

$\left\|x_{n+1}-x^{*}\right\|^{2} \leq\left\|y_{n}-x^{*}\right\|^{2}-2 \alpha_{n} \Phi\left(\left\|y_{n}-x^{*}\right\|\right)+2 \alpha_{n} M_{1} A_{n}$.

Taking (50) into (51) and taking (51) into (52),

$$
\begin{aligned}
\left\|x_{n+1}-x^{*}\right\|^{2} \leq & \left\|x_{n}-x^{*}\right\|^{2}-2 \gamma_{n} \Phi\left(\left\|x_{n}-x^{*}\right\|\right)+2 \gamma_{n} M_{1} C_{n} \\
& -2 \beta_{n} \Phi\left(\left\|z_{n}-x^{*}\right\|\right)-2 \alpha_{n} \Phi\left(\left\|y_{n}-x^{*}\right\|\right) \\
& +2 \beta_{n} M_{1} B_{n}+2 \alpha_{n} M_{1} A_{n} \\
\leq & \left\|x_{n}-x^{*}\right\|^{2}-2 \gamma_{n} \Phi\left(\left\|x_{n}-x^{*}\right\|\right)-2 \alpha_{n} \Phi\left(\left\|y_{n}-x^{*}\right\|\right) \\
& +2 \gamma_{n}\left[\frac{\alpha_{n}}{\gamma_{n}} M_{1} A_{n}+\frac{\beta_{n}}{\gamma_{n}} M_{1} B_{n}+M_{1} C_{n}\right] \\
& -2 \beta_{n} \Phi\left(\left\|z_{n}-x^{*}\right\|\right),
\end{aligned}
$$

where $Q_{n}=\left(\alpha_{n} / \gamma_{n}\right) M_{1} A_{n}+\left(\beta_{n} / \gamma_{n}\right) M_{1} B_{n}+M_{1} C_{n} \longrightarrow 0$ as $n \longrightarrow \infty$.

Set inf $\left(\Phi\left(\left\|z_{n}-x^{*}\right\|\right) / \Phi\left(\left\|x_{n}-x^{*}\right\|\right)+1\right)=W$; since $\Phi$ is a strictly increasing continuous function, then $W$ exists. Thus,

$$
\Phi\left(\left\|z_{n}-x^{*}\right\|\right) \geq W \Phi\left(\left\|x_{n}-x^{*}\right\|\right)+W \geq W \Phi\left(\left\|x_{n}-x^{*}\right\|\right) .
$$

Set inf $\left(\Phi\left(\left\|y_{n}-x^{*}\right\|\right) / \Phi\left(\left\|x_{n}-x^{*}\right\|\right)+1\right)=N$; since $\Phi$ is a strictly increasing continuous function, then $N$ exists. Thus,

$$
\Phi\left(\left\|y_{n}-x^{*}\right\|\right) \geq N \Phi\left(\left\|x_{n}-x^{*}\right\|\right)+N \geq N \Phi\left(\left\|x_{n}-x^{*}\right\|\right) .
$$

Then,

$$
\begin{aligned}
\left\|x_{n+1}-x^{*}\right\|^{2} \leq & \left\|x_{n}-x^{*}\right\|^{2}+2 \gamma_{n} Q_{n}-2 \gamma_{n} \Phi\left(\left\|x_{n}-x^{*}\right\|\right) \\
& -2 \beta_{n} W \Phi\left(\left\|x_{n}-x^{*}\right\|\right)-2 \alpha_{n} N \Phi\left(\left\|x_{n}-x^{*}\right\|\right) \\
\leq & \left\|x_{n}-x^{*}\right\|^{2}+2 \gamma_{n} Q_{n} \\
& -2 \gamma_{n} \Phi\left(\left\|x_{n}-x^{*}\right\|\right)\left[1+\frac{\beta_{n}}{\gamma_{n}} W+\frac{\alpha_{n}}{\gamma_{n}} N\right] .
\end{aligned}
$$

Let $\lambda_{n}=\left\|x_{n}-x^{*}\right\|^{2}, S_{n}=2\left(1+\left(\beta_{n} / \gamma_{n}\right) W+\left(\alpha_{n} / \gamma_{n}\right) N\right)$ and $\rho_{n}=2 \gamma_{n} Q_{n}$; then, from inequality (56), we obtain that $\lambda_{n+1} \leq \lambda_{n}-\gamma_{n} S_{n} \Phi\left(\lambda_{n}\right)+\rho_{n}$, where $\left(\rho_{n} / \gamma_{n} S_{n}\right) \longrightarrow 0 \quad$ as $n \longrightarrow \infty$. Therefore, the conclusion of the theorem follows from Proposition 4. Uniqueness of $x^{*}$ is derived from the definition of $T$.

By Definition 4, we know that the generalized Lipschitz mapping is the extension of nonexpansive mapping, so the following corollary follows trivially.

Corollary 3. Suppose D is a nonempty closed convex subset of a real uniformly smooth Banach space E. Suppose $T: D \longrightarrow D$ is a bounded generalized $\Phi$-hemi-contractive mapping and $x^{*} \in F(T) \neq \varnothing$. For arbitrary $x_{0} \in D,\left\{x_{n}\right\}$ be a $S P$ iterative sequence defined by (4), where $\left\{\alpha_{n}\right\}$, $\left\{\beta_{n}\right\},\left\{\gamma_{n}\right\} \subseteq[0,1], \lim _{n \longrightarrow \infty} \alpha_{n}=\lim _{n \longrightarrow \infty} \beta_{n}=0, \gamma_{n}=o\left(\beta_{n}\right)$, and $\sum \gamma_{n}=\infty$. Then, there exists a constant $d_{0}>0$ such that if $0<\alpha_{n}, \beta_{n}, \gamma_{n} \leq d_{0},\left\{x_{n}\right\}$ converges strongly to the unique fixed point $x^{*}$ of $T$.

The following corollary follows trivially from Definition 2 and Definition 3.

Corollary 4. Suppose $E$ is a real uniformly smooth Banach space. Suppose $T: E \longrightarrow E$ is a bounded generalized $\Phi$-accretive mapping and the solution $x^{*}$ of the equation $T x=0$ exists. For arbitrary $x_{0} \in E,\left\{x_{n}\right\}$ in $E$ is defined as

$$
\left\{\begin{array}{l}
x_{n+1}=\left(1-\alpha_{n}\right) y_{n}-\alpha_{n} T y_{n}, \\
y_{n}=\left(1-\beta_{n}\right) z_{n}-\beta_{n} T z_{n}, \\
z_{n}=\left(1-\gamma_{n}\right) x_{n}-\gamma_{n} T x_{n}, \quad n \geq 0,
\end{array}\right.
$$

where $\left\{\alpha_{n}\right\},\left\{\beta_{n}\right\},\left\{\gamma_{n}\right\} \subseteq[0,1], \lim _{n \longrightarrow \infty} \alpha_{n}=\lim _{n \longrightarrow \infty} \beta_{n}=0$, $\gamma_{n}=o\left(\beta_{n}\right)$, and $\sum \gamma_{n}=\infty$. Then, there exists a constant $d_{0}>0$ such that if $0<\alpha_{n}, \beta_{n}, \gamma_{n} \leq d_{0},\left\{x_{n}\right\}$ converges strongly to the unique solution $T x=0$.

\section{Experiments}

In this section, we analyze the rate of convergence of four iterative schemes, namely, Noor iteration, iteration of 


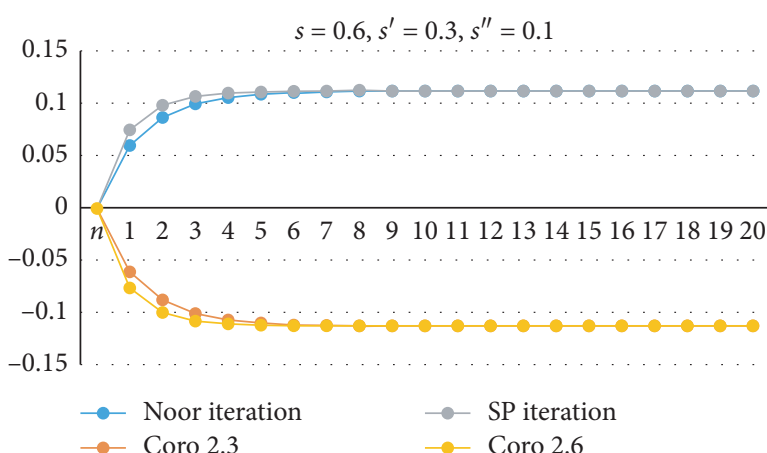

(a)

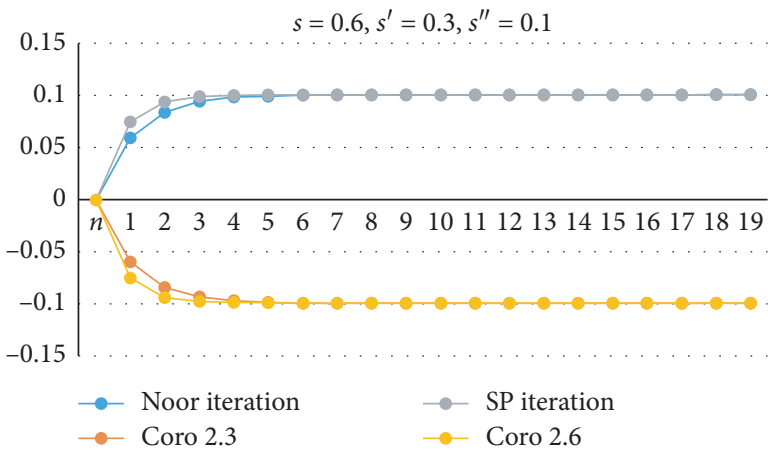

(c)

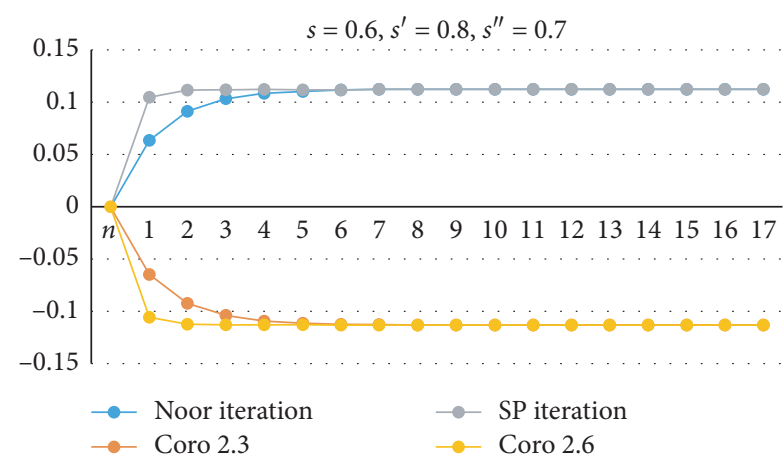

(b)

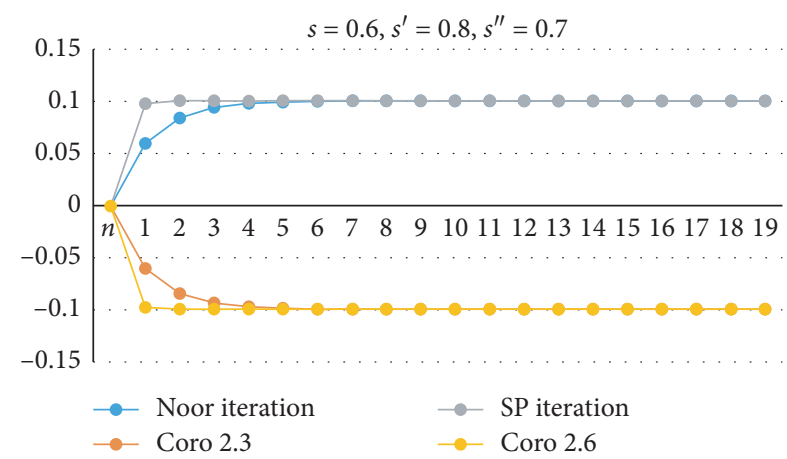

(d)

Figure 1: (a, b) Graphs of quadratic functions in Tables 1 and 2, respectively. (c, d) Graphs of cubic functions in Tables 3 and 4, respectively.

TABLE 1: Iterations of quadratic function $s=0.6, s^{\prime}=0.3, s^{\prime \prime}=0.1$.

\begin{tabular}{lcccc}
\hline & & \multicolumn{2}{c}{$s=0.6, s^{\prime}=0.3, s^{\prime \prime}=0.1$} & SP \\
$n$ & Noor & Co2.3 & $F(x)$ & -0.6 \\
\hline 1 & $F(x)$ & $F(x)$ & 0.0756347 & -0.0756347 \\
2 & 0.0605411 & -0.0605411 & 0.0992939 & -0.0992939 \\
3 & 0.0874702 & -0.0874702 & 0.107705 & -0.107705 \\
4 & 0.100248 & -0.100248 & 0.11082 & -0.11082 \\
5 & 0.106495 & -0.106495 & 0.11199 & -0.11199 \\
6 & 0.109594 & -0.109594 & 0.112432 & -0.112432 \\
7 & 0.111142 & -0.111142 & 0.1126 & -0.1126 \\
8 & 0.111918 & -0.111918 & 0.112663 & -0.112687 \\
9 & 0.112308 & -0.112308 & 0.112687 & -0.112696 \\
10 & 0.112503 & -0.112503 & 0.112696 & -0.1127 \\
11 & 0.112602 & -0.112602 & 0.1127 & -0.112701 \\
12 & 0.112652 & -0.112652 & 0.112701 & -0.112701 \\
13 & 0.112676 & -0.112676 & 0.112701 & $-\mathbf{0 . 1 1 2 7 0 2}$ \\
14 & 0.112689 & -0.112689 & $\mathbf{0 . 1 1 2 7 0 2}$ & -0.112702 \\
15 & 0.112695 & -0.112695 & 0.112702 & -0.112702 \\
16 & 0.112698 & -0.112698 & 0.112702 & -0.112702 \\
17 & 0.1127 & -0.1127 & 0.112702 & -0.112702 \\
18 & 0.112701 & -0.112701 & 0.112702 & -0.112702 \\
19 & 0.112701 & -0.112701 & 0.112702 & $\ldots$ \\
20 & 0.112701 & -0.112701 & 0.112702 & $\ldots$ \\
$\ldots$ & $\mathbf{0 . 1 1 2 7 0 2}$ & $\ldots .112702$ & $\mathbf{0 . 1 1 2 7 0 2}$ & -0.112702 \\
25 & $\ldots$ & $-\mathbf{0 . 1 1 2 7 0 2}$ & $-\mathbf{0 . 1 1 2 7 0 2}$ \\
\hline
\end{tabular}


TABLE 2: Iterations of quadratic function $s=0.6, s^{\prime}=0.8, s^{\prime \prime}=0.7$.

\begin{tabular}{|c|c|c|c|c|}
\hline \multirow[b]{2}{*}{$n$} & \multicolumn{4}{|c|}{$s=0.6, s^{\prime}=0.8, s^{\prime \prime}=0.7$} \\
\hline & $\begin{array}{l}\text { Noor } \\
F(x)\end{array}$ & $\begin{array}{l}\text { Co } 2.3 \\
F(x)\end{array}$ & $\begin{array}{c}\text { SP } \\
F(x)\end{array}$ & $\begin{array}{c}\text { Co } 2.6 \\
F(x)\end{array}$ \\
\hline 1 & 0.0642255 & -0.0642255 & 0.104921 & -0.104921 \\
\hline 2 & 0.091647 & -0.091647 & 0.111992 & -0.111992 \\
\hline 3 & 0.103513 & -0.103513 & 0.112636 & -0.112636 \\
\hline 4 & 0.108682 & -0.108682 & 0.112696 & -0.112696 \\
\hline 5 & 0.110942 & -0.110942 & 0.112701 & -0.112701 \\
\hline 6 & 0.111931 & -0.111931 & 0.112702 & -0.112702 \\
\hline 7 & 0.112364 & -0.112364 & 0.112702 & -0.112702 \\
\hline 8 & 0.112554 & -0.112554 & 0.112702 & -0.112702 \\
\hline 9 & 0.112637 & -0.112637 & 0.112702 & -0.112702 \\
\hline 10 & 0.112673 & -0.112673 & 0.112702 & -0.112702 \\
\hline 11 & 0.112689 & -0.112689 & 0.112702 & -0.112702 \\
\hline 12 & 0.112696 & -0.112696 & 0.112702 & -0.112702 \\
\hline 13 & 0.112699 & -0.112699 & 0.112702 & -0.112702 \\
\hline 14 & 0.112701 & -0.112701 & 0.112702 & -0.112702 \\
\hline 15 & 0.112701 & -0.112701 & 0.112702 & -0.112702 \\
\hline 16 & 0.112701 & -0.112701 & 0.112702 & -0.112702 \\
\hline 17 & 0.112702 & -0.112702 & 0.112702 & -0.112702 \\
\hline $\begin{array}{l}\cdots \\
25\end{array}$ & $\begin{array}{c}\cdots \\
0.112702\end{array}$ & $\begin{array}{c}\cdots \\
-0.112702\end{array}$ & 0.112702 & -0.112702 \\
\hline
\end{tabular}

TABLE 3: Iterations of cubic function $s=0.6, s^{\prime}=0.3, s^{\prime \prime}=0.1$.

\begin{tabular}{|c|c|c|c|c|}
\hline \multirow[b]{2}{*}{$n$} & \multicolumn{4}{|c|}{$s=0.6, s^{\prime}=0.3, s^{\prime \prime}=0.1$} \\
\hline & $\begin{array}{l}\text { Noor } \\
F(x)\end{array}$ & $\begin{array}{l}\mathrm{Co} 2.3 \\
F(x)\end{array}$ & $\begin{array}{c}\text { SP } \\
F(x)\end{array}$ & $\begin{array}{l}\mathrm{Co} 2.6 \\
F(x)\end{array}$ \\
\hline 1 & 0.0600162 & -0.0599838 & 0.0748305 & -0.0747695 \\
\hline 2 & 0.0842313 & -0.0837704 & 0.0940842 & -0.0932201 \\
\hline 3 & 0.0941177 & -0.0930929 & 0.0991753 & -0.0976483 \\
\hline 4 & 0.0981802 & -0.0967241 & 0.100534 & -0.0987014 \\
\hline 5 & 0.0998545 & -0.0981347 & 0.100898 & -0.0989512 \\
\hline 6 & 0.100545 & -0.098682 & 0.100996 & -0.0990104 \\
\hline 7 & 0.100831 & -0.0988944 & 0.101022 & -0.0990245 \\
\hline 8 & 0.100948 & -0.0989767 & 0.101029 & -0.0990278 \\
\hline 9 & 0.100997 & -0.0990086 & 0.101031 & -0.0990286 \\
\hline 10 & 0.101017 & -0.099021 & 0.101031 & -0.0990288 \\
\hline 11 & 0.101025 & -0.0990258 & 0.101031 & -0.0990288 \\
\hline 12 & 0.101029 & -0.0990277 & 0.101031 & -0.0990288 \\
\hline 13 & 0.10103 & -0.0990284 & 0.101031 & -0.0990289 \\
\hline 14 & 0.101031 & -0.0990287 & 0.101031 & -0.0990289 \\
\hline 15 & 0.101031 & -0.0990288 & 0.101031 & -0.0990289 \\
\hline 16 & 0.101031 & -0.0990288 & 0.101031 & -0.0990289 \\
\hline 17 & 0.101031 & -0.0990288 & 0.101031 & -0.0990289 \\
\hline 18 & 0.101031 & -0.0990288 & 0.101031 & -0.0990289 \\
\hline 19 & 0.101031 & -0.0990289 & 0.101031 & -0.0990289 \\
\hline$\cdots$ & 0.101031 & -0.0990289 & 0.101031 & -0.0990289 \\
\hline
\end{tabular}

Corollary 2, SP iteration, and iteration of Corollary 4, iterative schemes for complex space by using Visual Studio. The results obtained are extensions of some recent results of Rana et al. [15] and Chugh et al. [16].

We take $\alpha_{n}=s, \beta_{n}=s^{\prime}$, and $\gamma_{n}=s^{\prime \prime}$ and derive the fixed points of the following polynomial functions:

$$
\text { Quadratic functions }=z^{2}+c
$$

Cubic functions $=z^{3}+c$

Biquadratic functions $=z^{4}+c$

Recently, Rana et al. [15] drew a comparative analysis of Picard, Mann, and Ishikawa iterative schemes by starting with $z=(0,0)$ and $c=0.1$ in complex space. In this paper, we will continue the comparative study in complex space by taking the same $z$ and $c$, for Noor iteration, iteration of 
TABLE 4: Iterations of cubic function $s=0.6, s^{\prime}=0.8, s^{\prime \prime}=0.7$.

\begin{tabular}{|c|c|c|c|c|}
\hline \multirow[b]{2}{*}{$n$} & \multicolumn{4}{|c|}{$s=0.6, s^{\prime}=0.8, s^{\prime \prime}=0.7$} \\
\hline & $\begin{array}{l}\text { Noor } \\
F(x)\end{array}$ & $\begin{array}{c}\text { Co } 2.3 \\
F(x)\end{array}$ & $\begin{array}{c}\text { SP } \\
F(x)\end{array}$ & $\begin{array}{c}\text { Co } 2.6 \\
F(x)\end{array}$ \\
\hline 1 & 0.0603104 & -0.059696 & 0.0982125 & -0.0969962 \\
\hline 2 & 0.0846007 & -0.0834203 & 0.100946 & -0.0989904 \\
\hline 3 & 0.0943985 & -0.0928372 & 0.101029 & -0.0990281 \\
\hline 4 & 0.0983532 & -0.0965731 & 0.101031 & -0.0990288 \\
\hline 5 & 0.0999499 & -0.0980549 & 0.101031 & -0.0990289 \\
\hline 6 & 0.100595 & -0.0986426 & 0.101031 & -0.0990289 \\
\hline 7 & 0.100855 & -0.0988757 & 0.101031 & -0.0990289 \\
\hline 8 & 0.10096 & -0.0989681 & 0.101031 & -0.0990289 \\
\hline 9 & 0.101003 & -0.0990048 & 0.101031 & -0.0990289 \\
\hline 10 & 0.10102 & -0.0990193 & 0.101031 & -0.0990289 \\
\hline 11 & 0.101027 & -0.0990251 & 0.101031 & -0.0990289 \\
\hline 12 & 0.101029 & -0.0990273 & 0.101031 & -0.0990289 \\
\hline 13 & 0.10103 & -0.0990283 & 0.101031 & -0.0990289 \\
\hline 14 & 0.101031 & -0.0990286 & 0.101031 & -0.0990289 \\
\hline 15 & 0.101031 & -0.0990288 & 0.101031 & -0.0990289 \\
\hline 16 & 0.101031 & -0.0990288 & 0.101031 & -0.0990289 \\
\hline 17 & 0.101031 & -0.0990288 & 0.101031 & -0.0990289 \\
\hline 18 & 0.101031 & -0.0990288 & 0.101031 & -0.0990289 \\
\hline 19 & 0.101031 & -0.0990289 & 0.101031 & -0.0990289 \\
\hline$\ldots$ & $\ldots$ & $\ldots$ & $\ldots$ & $\ldots$ \\
\hline 25 & 0.101031 & -0.0990289 & 0.101031 & -0.0990289 \\
\hline
\end{tabular}

TABLE 5: Iterations of biquadratic function $s=0.6, s^{\prime}=0.1, s^{\prime \prime}=0.1$.

\begin{tabular}{lcccc}
\hline & & \multicolumn{2}{c}{$s=0.6, s^{\prime}=0.1, s^{\prime \prime}=0.1$} & \\
& Noor & Co2.3 & SP & $F(x)$ \\
$n$ & $F(x)$ & $F(x)$ & 0.0676001 & -0.0676001 \\
\hline 1 & 0.06 & -0.06 & 0.0895219 & -0.0895219 \\
2 & 0.0840101 & -0.0840101 & 0.0966522 & -0.0966522 \\
3 & 0.0936363 & -0.0936363 & 0.0989758 & -0.0989758 \\
4 & 0.0975019 & -0.0975019 & 0.0997335 & -0.0997335 \\
5 & 0.0990556 & -0.0990556 & 0.0999807 & -0.0999807 \\
6 & 0.0996802 & -0.0996802 & 0.100061 & -0.100061 \\
7 & 0.0999314 & -0.0999314 & 0.100088 & -0.100088 \\
8 & 0.100032 & -0.100032 & 0.100096 & -0.100096 \\
9 & 0.100073 & -0.100073 & 0.100099 & -0.100099 \\
10 & 0.100089 & -0.100089 & $\mathbf{0 . 1 0 0 1}$ & $-\mathbf{0 . 1 0 0 1}$ \\
11 & 0.100096 & -0.100096 & 0.1001 & -0.1001 \\
12 & 0.100099 & -0.100099 & 0.1001 & -0.1001 \\
13 & $\mathbf{0 . 1 0 0 1}$ & $-\mathbf{0 . 1 0 0 1}$ & $\ldots$ & $\ldots$ \\
$\ldots$ & $\ldots$ & $\ldots$ & $\mathbf{0 . 1 0 0 1}$ & $-\mathbf{0 . 1 0 0 1}$ \\
\hline
\end{tabular}

Corollary 2, SP iteration, and iteration of Corollary 4 and, hence, extend the results of Rana et al. [15] and Chugh et al. [16].

Quadratic functions are provided in Tables 1 and 2, and their corresponding graphs are shown in Figures 1(a) and 1(b), respectively. Cubic functions are provided in Tables 3 and 4, and their corresponding graphs are shown in Figures 1(c) and 1(d). Biquadratic functions are provided in Tables 5-8, and their corresponding graphs are shown in Figures 1(a)-1(d). 
TABLE 6: Iterations of biquadratic function $s=0.6, s^{\prime}=0.3, s^{\prime \prime}=0.1$.

\begin{tabular}{|c|c|c|c|c|}
\hline \multirow[b]{2}{*}{$n$} & \multicolumn{4}{|c|}{$s=0.6, s^{\prime}=0.3, s^{\prime \prime}=0.1$} \\
\hline & $\begin{array}{l}\text { Noor } \\
F(x)\end{array}$ & $\begin{array}{l}\mathrm{Co} 2.3 \\
F(x)\end{array}$ & $\begin{array}{c}\text { SP } \\
F(x)\end{array}$ & $\begin{array}{c}\text { Co2.6 } \\
F(x)\end{array}$ \\
\hline 1 & 0.0600005 & -0.0600005 & 0.0748011 & -0.0748011 \\
\hline 2 & 0.0840163 & -0.0840163 & 0.0936851 & -0.0936851 \\
\hline 3 & 0.0936439 & -0.0936439 & 0.0984714 & -0.0984714 \\
\hline 4 & 0.0975076 & -0.0975076 & 0.0996866 & -0.0996866 \\
\hline 5 & 0.099059 & -0.099059 & 0.0999953 & -0.0999953 \\
\hline 6 & 0.0996821 & -0.0996821 & 0.100074 & -0.100074 \\
\hline 7 & 0.0999324 & -0.0999324 & 0.100094 & -0.100094 \\
\hline 8 & 0.100033 & -0.100033 & 0.100099 & -0.100099 \\
\hline 9 & 0.100073 & -0.100073 & 0.1001 & -0.1001 \\
\hline 10 & 0.10009 & -0.10009 & 0.1001 & -0.1001 \\
\hline 11 & 0.100096 & -0.100096 & 0.1001 & -0.1001 \\
\hline 12 & 0.100099 & -0.100099 & 0.1001 & -0.1001 \\
\hline 13 & 0.1001 & -0.1001 & 0.1001 & -0.1001 \\
\hline$\cdots$ & $\ldots$ & $\cdots$ & $\ldots$ & $\ldots$ \\
\hline 25 & 0.1001 & -0.1001 & 0.1001 & -0.1001 \\
\hline
\end{tabular}

TABLE 7: Iterations of biquadratic function $s=0.6, s^{\prime}=0.5, s^{\prime \prime}=0.4$.

\begin{tabular}{lcccc}
\hline & & \multicolumn{2}{c}{$s=0.6, s^{\prime}=0.5, s^{\prime \prime}=0.4$} & \\
& Noor & Co2.3 & $F(x)$ & $F(x)$ \\
$n$ & $F(x)$ & $F(x)$ & 0.0880149 & -0.0880149 \\
1 & 0.0600038 & -0.0600038 & 0.0986334 & -0.0986334 \\
2 & 0.0840261 & -0.0840261 & 0.0999221 & -0.0999221 \\
3 & 0.0936535 & -0.0936535 & 0.100079 & -0.100079 \\
4 & 0.0975142 & -0.0975142 & 0.100098 & -0.100098 \\
5 & 0.0990629 & -0.0990629 & $\mathbf{0 . 1 0 0 1}$ & $-\mathbf{0 . 1 0 0 1}$ \\
6 & 0.0996841 & -0.0996841 & 0.1001 & -0.1001 \\
7 & 0.0999334 & -0.0999334 & 0.1001 & -0.1001 \\
8 & 0.100033 & -0.100033 & 0.1001 & -0.1001 \\
9 & 0.100074 & -0.100074 & 0.1001 & -0.1001 \\
10 & 0.10009 & -0.10009 & 0.1001 & -0.1001 \\
11 & 0.100096 & -0.100096 & 0.1001 & -0.1001 \\
12 & 0.100099 & -0.100099 & 0.1001 & -0.1001 \\
13 & $\mathbf{0 . 1 0 0 1}$ & $-\mathbf{0 . 1 0 0 1}$ & $\ldots$ & $\ldots$ \\
$\ldots$ & $\ldots$ & $\ldots$ & $\mathbf{0 . 1 0 0 1}$ & $-\mathbf{0 . 1 0 0 1}$ \\
25 & $\mathbf{0 . 1 0 0 1}$ & $-\mathbf{0 . 1 0 0 1}$ & & \\
\hline
\end{tabular}

TABLE 8: Iterations of biquadratic function $s=0.6, s^{\prime}=0.8, s^{\prime \prime}=0.7$.

\begin{tabular}{lcccc}
\hline & & \multicolumn{2}{c}{$s=0.6, s^{\prime}=0.8, s^{\prime \prime}=0.7$} & SP \\
$n$ & Noor & Co2.3 & $F(x)$ & $F(x)$ \\
\hline 1 & $F(x)$ & $F(x)$ & 0.0976546 & -0.0976546 \\
2 & 0.0600246 & -0.0600246 & 0.10004 & -0.10004 \\
3 & 0.0840529 & -0.0840529 & 0.100099 & -0.100099 \\
4 & 0.093674 & -0.093674 & $\mathbf{0 . 1 0 0 1}$ & $-\mathbf{0 . 1 0 0 1}$ \\
5 & 0.0975268 & -0.0975268 & 0.1001 & -0.1001 \\
6 & 0.0990697 & -0.0990697 & 0.1001 & -0.1001 \\
7 & 0.0996876 & -0.0996876 & 0.1001 & -0.1001 \\
8 & 0.0999351 & -0.0999351 & 0.1001 & -0.1001 \\
9 & 0.100034 & -0.100034 & 0.1001 & -0.1001 \\
10 & 0.100074 & -0.100074 & 0.1001 & -0.1001 \\
11 & 0.10009 & -0.10009 & 0.1001 & -0.1001 \\
12 & 0.100096 & -0.100096 & 0.1001 & -0.1001 \\
13 & 0.100099 & -0.100099 & 0.1001 & -0.1001 \\
$\ldots$ & $\mathbf{0 . 1 0 0 1}$ & $-\mathbf{0 . 1 0 0 1}$ & $\ldots$ & $\ldots$ \\
25 & $\ldots$ & $\ldots$ & $\mathbf{0 . 1 0 0 1}$ \\
\hline
\end{tabular}




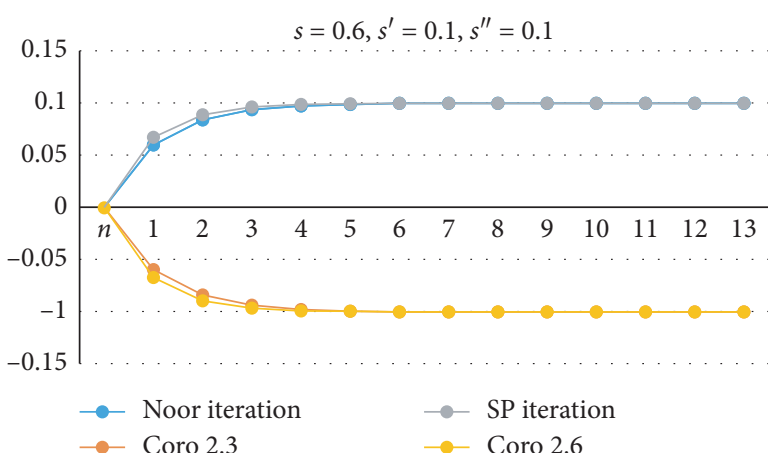

(a)

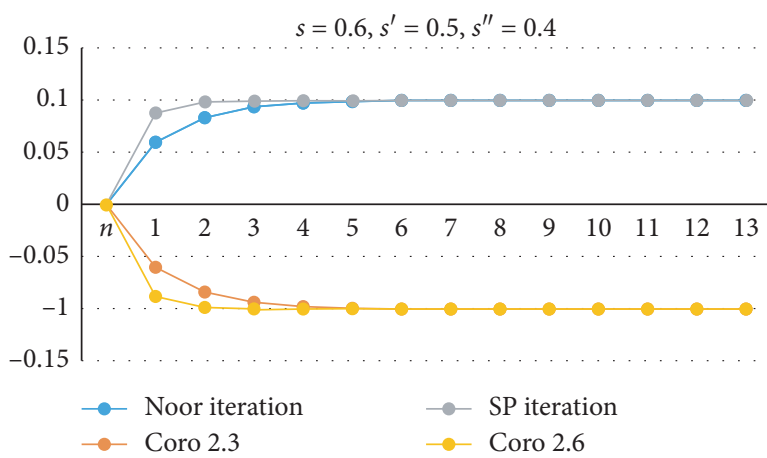

(c)

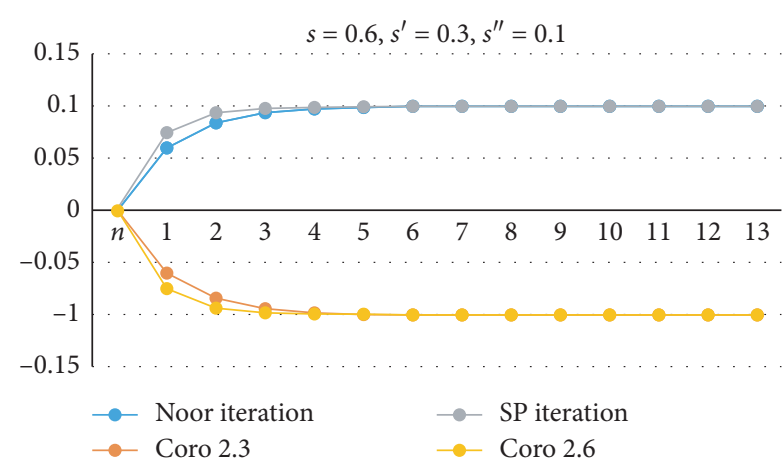

(b)

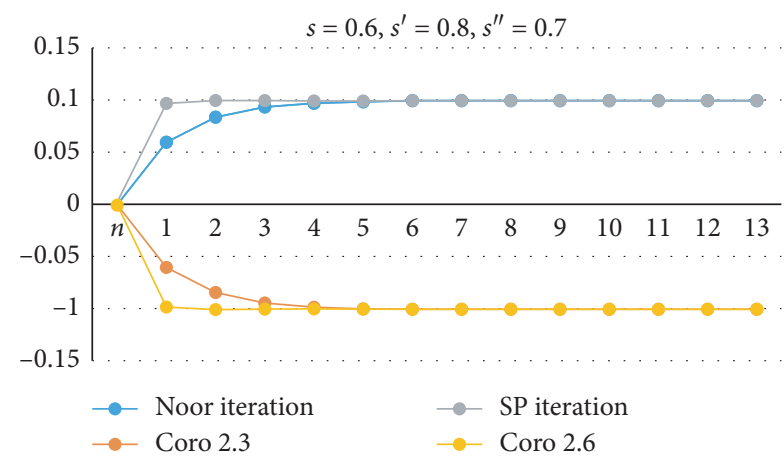

(d)

Figure 2: (a-d) Graphs of biquadratic functions given in Tables 5-8, respectively.

\section{Conclusion}

Keeping in mind comparative analysis drawn by $[15,16]$, Tables $1-8$, we conclude that

(i) In case of quadratic polynomial, $F(x)$ of Noor and Co2.3 iterative schemes and SP and Co2.6 iterative schemes are opposite to each other; they show equivalence,and the speed of convergence of iterative schemes is compared as follows: SP > Noor; Co2.6>Co2.3.

(ii) In case of cubic polynomial, the speed of convergence of iterative schemes is compared as follows: $\mathrm{SP}>$ Noor; Co2.6 > Co2.3; Noor > Co2.3; SP > Co2.6.

(iii) In case of biquadratic polynomial, $F(x)$ of Noor and Co2.3 iterative schemes and SP and Co2.6 iterative schemes are opposite to each other; they show equivalence, and the speed of convergence of iterative schemes is compared as follows: SP > Noor; Co2.6 > Co2.3 (Figure 2).

(iv) In the case of biquadratic polynomial, the most important discovery is that, as long as the value of $s$ is set, the convergence rate of Noor and the convergence rate of Co2.3 iterative schemes will not change.

\section{Data Availability}

The data used to support the findings of this study are included within the article and are available from the corresponding author upon request.

\section{Conflicts of Interest}

The authors declare that they have no conflicts of interest.

\section{Acknowledgments}

This work was supported by Applied Basic Research Foundation of Sichuan Province of China (Grant no. 2018JY0169).

\section{References}

[1] C. Charles, Geometric Properties of Banach Spaces and Nonlinear Iterations, Springer, Berlin, Germany, 2009.

[2] M. A. Noor, T. M. Rassias, and Z. Huang, "Three-step iterations for accretive operator equations," Journal of Mathematical Analysis and Applications, vol. 274, pp. 59-68, 2002.

[3] X. Qin and J. C. Yao, "Weak convergence of a Mann-like algorithm for nonexpansive and accretive operators," Journal of Inequalities and Applications, vol. 2016, p. 232, 2016.

[4] B.-S. Lee, "Strong convergence theorems with a Noor-type iterative scheme in convex metric spaces," Computers and Mathematics with Applications, vol. 61, pp. 3218-3225, 2011.

[5] Y. G. Xu, "Ishikawa and Mann iterative processes with errors for nonlinear strongly accretive operator equations," Journal of Mathematical Analysis and Applications, vol. 224, pp. 91101, 1998.

[6] M. A. Noor, "New approximation schemes for general variational inequalities," Journal of Mathematical Analysis and Applications, vol. 251, pp. 217-229, 2000.

[7] M. A. Noor, "Three-step iterative algorithms for multi-valued quasi variational inclusions," Journal of Mathematical Analysis and Applications, vol. 255, pp. 589-604, 2001. 
[8] W. Phuengrattana and S. Suantai, "On the rate of convergence of Mann, Ishikawa, Noor and SP-iterations for continuous functions on an arbitrary interval," Journal of Computational and Applied Mathematics, vol. 235, no. 9, pp. 3006-3014, 2011.

[9] D. F. Cudia, "The geometry of Banach spaces. Smoothness," Transactions of the American Mathematical Society, vol. 110, no. 2, p. 284, 1964.

[10] X. Qin, L. Wang, and J. C. Yao, "Inertial splitting method for maximal monotone mappings," Journal of Nonlinear and Convex Analysis, vol. 21, pp. 2325-2333, 2020.

[11] F. E. Browder, "Nonlinear operators and nonlinear equations of evolution in Banach spaces," in Proceedings of Symposia in Pure Mathematics XVIII, Providence, RI, USA, November 1976.

[12] Y. Shehu and J. C. Yao, "Rate of convergence for inertial iterative method for countable family of certain quasi-nonexpansive mappings," Journal of Nonlinear and Convex Analysis, vol. 21, pp. 533-541, 2020.

[13] H. Y. Zhou and D. Q. Chen, "Iterative approximations of fixed points for nonlinear mappings of $\Phi$-hemi-contractive type in normed linear spaces," Mathematical Applications, vol. 11, no. 3, pp. 118-121, 1998.

[14] Z. Q. Xue, H. Y. Zhou, and Y. J. Cho, "Iterative solutions of nonlinear equations for $\mathrm{m}$-accretive operators in Banach space," Journal of Nonlinear and Convex Analysis, vol. 1, no. 3, pp. 313-320, 2003.

[15] R. Rana, R. C. Dimri, and A. Tomar, "Remarks on convergence among Picard, Mann and Ishikawa iteration for complex space," International Journal of Computer Applications, vol. 21, no. 9, pp. 0975-8887, 2011.

[16] R. Chugh, V. Kumar, and O. Dahiya, "Comparative analysis of rate of convergence of Agarwal et al., Noor and SP iterative schemes for complex space," International Journal of Computer Applications, vol. 41, pp. 0975-8887, 2012. 